\title{
Geometry Types for Graphics Programming
}

\author{
DIETRICH GEISLER, Cornell University, United States
}

IRENE YOON, University of Pennsylvania, United States

ADITI KABRA, Carnegie Mellon University, United States

HORACE HE, YINNON SANDERS, and ADRIAN SAMPSON, Cornell University, United States

In domains that deal with physical space and geometry, programmers need to track the coordinate systems that underpin a computation. We identify a class of geometry bugs that arise from confusing which coordinate system a vector belongs to. These bugs are not ruled out by current languages for vector-oriented computing, are difficult to check for at run time, and can generate subtly incorrect output that can be hard to test for.

We introduce a type system and language that prevents geometry bugs by reflecting the coordinate system for each geometric object. A value's geometry type encodes its reference frame, the kind of geometric object (such as a point or a direction), and the coordinate representation (such as Cartesian or spherical coordinates) We show how these types can rule out geometrically incorrect operations, and we show how to use them to automatically generate correct-by-construction code to transform vectors between coordinate systems. We implement a language for graphics programming, Gator, that checks geometry types and compiles to OpenGL's shading language, GLSL. Using case studies, we demonstrate that Gator can raise the level of abstraction for shader programming and prevent common errors without inducing significant annotation overhead or performance cost.

CCS Concepts: • Computing methodologies $\rightarrow$ Computer graphics; $\bullet$ Software and its engineering $\rightarrow$ Data types and structures.

Additional Key Words and Phrases: computer graphics, language design, type systems, geometry

\section{ACM Reference Format:}

Dietrich Geisler, Irene Yoon, Aditi Kabra, Horace He, Yinnon Sanders, and Adrian Sampson. 2020. Geometry Types for Graphics Programming. Proc. ACM Program. Lang. 4, OOPSLA, Article 173 (November 2020), 25 pages. https://doi.org/10.1145/3428241

\section{INTRODUCTION}

Applications across a broad swath of domains use linear algebra to represent geometry, coordinates, and simulations of the physical world. Scientific computing workloads, robotics control software, and real-time graphics renderers all use matrices and vectors pervasively to manipulate points according to linear-algebraic laws. The programming languages that express these computations, however, rarely capture the underlying geometric properties of these operations. In domains where performance is critical, most languages provide only thin abstractions over the low-level vector and matrix data types that the underlying hardware (i.e., GPU) implements. A typical language might have a basic vec2 data type for vectors consisting of two floating-point numbers, for example,

Authors' addresses: Dietrich Geisler, dag368@cornell.edu, Cornell University, Ithaca, New York, United States, 14853; Irene Yoon, ey222@cornell.edu, University of Pennsylvania, Philadelphia, Pennsylvania, United States, 19104; Aditi Kabra, akabra@cs.cmu.edu, Carnegie Mellon University, Pittsburgh, Pennsylvania, United States, 15213; Horace He, hh498@cornell. edu; Yinnon Sanders, yys4@cornell.edu; Adrian Sampson, asampson@cs.cornell.edu, Cornell University, Ithaca, New York, United States, 14853.

This work is licensed under a Creative Commons Attribution 4.0 International License.

(c) 2020 Copyright held by the owner/author(s).

2475-1421/2020/11-ART173

https://doi.org/10.1145/3428241

Proc. ACM Program. Lang., Vol. 4, No. OOPSLA, Article 173. Publication date: November 2020. 


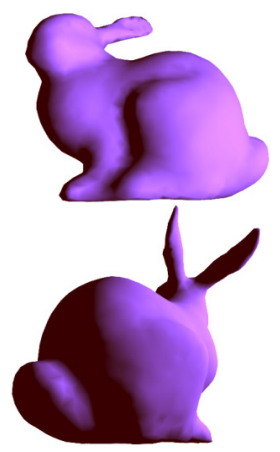

(a) Correct implementation.

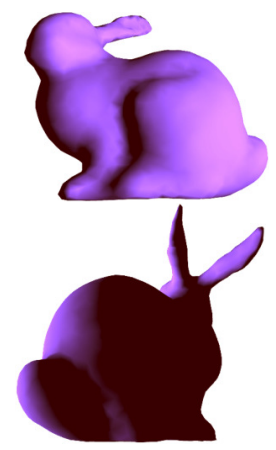

(b) With geometry bug.

Fig. 1. Objects rendered with an implementation of the diffuse component of Phong lighting [Phong 1975], without (a) and with (b) a coordinate system transformation bug. The root cause is an incorrect spatial translation of the light source. The problem is only visible from one side of the model.

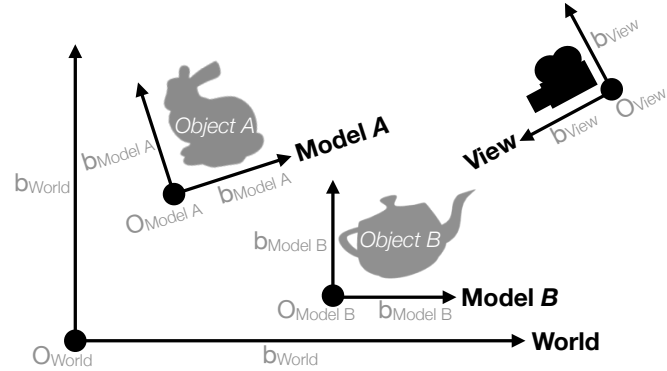

Fig. 2. Coordinate systems in graphics code. Model A, Model B, World, and View are coordinate systems. A coordinate system is defined by its basis vectors $b$ and origin $O$. The View represents the perspective of a simulated camera.

but not distinguish between 2D vectors in rectangular or polar coordinates-or between points in differently scaled rectangular coordinate systems.

This paper focuses on real-time 3D rendering on GPUs, where correctness hazards in linear algebra code are particularly pervasive. The central problem is that graphics code frequently entangles application logic with abstract geometric reasoning. Programmers must juggle vectors from a multitude of distinct coordinate systems while simultaneously optimizing for performance. This conflation of abstraction and implementation concerns makes it easy to confuse different coordinate system representations and to introduce subtle bugs. Figure 1 shows an example: a coordinate system bug yields incorrect visual output that would be difficult to catch with testing.

\subsection{The Problem}

Coordinate systems proliferate in graphics programming because 3D scenes consist of many individual objects. Figure 2 depicts a standard setup for rendering two objects in a single scene. Each object comes specified as a mesh, which consists of coordinate vectors for each vertex position. The mesh provides these vectors in a local, object-specific coordinate system called model space. The application positions multiple objects relative to one another in world space, and the simulated camera's position and angle define a view space.

Renderer code needs to combine vectors from different coordinate systems, such as in this distance calculation:

float dist $=$ length (teapotVertex - bunnyVertex $)$;

This code may be incorrect, however, depending on the representation of the teapotVertex and bunnyVertex vectors. If the values come from the mesh data, they are each represented in their respective model spaces-and subtracting them yields a geometrically meaningless result. A correct computation needs to convert the operands into a common coordinate system using affine transformation matrices:

float dist $=$ length (teapotToWorld $*$ teapotVertex - bunnyToWorld $*$ bunnyVertex $) ;$ 
Here, the teapotToWorld and bunnyToWorld matrices define the transformations from each respective model space into world space.

Geometry bugs are hard to catch. Mainstream rendering languages like OpenGL's GLSL [Segal and Akeley 2017] cannot statically rule out coordinate system mismatches. In GLSL, the variables teapotVertex and bunnyVertex would both have the type vec3, i.e., a tuple of three floating-point numbers. These bugs are also hard to detect dynamically. They do not crash programs-they only manifest in visual blemishes. While the buggy output in Figure $1 \mathrm{~b}$ clearly differs from the correct output in Figure 1a, it can be unclear what has gone wrong-or, when examining the buggy output alone, that anything has gone wrong at all. The intended behavior of the bunny is that the model should rotate relative to the camera and the light-the effect of the bug is that the light source "follows" the model, so the lighting reflection always appears at the same place on the bunny's surface. In the correct version, on the other hand, the second angle of the bunny shows that the bunny has rotated without either the camera or light moving.

Writing unit tests or assertions to catch this kind of bug can be challenging: specifying behavior of a graphics program requires formalizing how the resulting scene should be perceived. Viewers can perceive many possible outputs as visually indistinguishable, so even an informal specification of what makes a renderer "correct," for documentation or testing, can be difficult to write.

Geometry bugs in the wild. Even among established graphics libraries, geometry bugs can remain latent until a seemingly benign API change reveals the bug. For example, in LOVR, a framework for rapidly building VR experiences, the developers discovered a bug where a variable that was in one space was being used as if it was in another. ${ }^{1}$ This bug lay dormant until, to quote one of the maintainers, "there was a change in the rendering method that amplified the problems caused by [the geometry bug]." The maintainer then noted that they needed to "go backfill this fix to all the docs/examples that have the broken version." Because their effects are hard to detect, geometry bugs can persist and cause subtle inaccuracies that grow as code evolves.

We found similar issues that arise when APIs fail to specify information about vector spaces. In the Processing graphical IDE, for example, confusion surrounding a camera API led to a 20 -comment thread before a developer concluded that "better documentation could alleviate this to some extent: it needs to be clear that modelspace is relative to the camera at the time of construction." ${ }^{2}$ Finally, in the visualization library GLVisualize.jl, users disagree about the space that the library uses for a light position. ${ }^{3}$ The root cause in both cases is that the programming language affords no opportunity to convey vector space information.

This paper advocates for making geometric spaces manifest in programs themselves via a type system. Language support for geometric spaces can remove ambiguity and provide self-documenting interfaces between parts of a program. Static type checking can automatically enforce preconditions on geometric operations that would otherwise be left unchecked.

\subsection{Geometry Types}

We introduce a type system that can eliminate this class of bugs, and we describe a mechanism for automatic transformation that can rule out some of them by construction. Geometry types describe the coordinate system representing each value and the transformations that manipulate them. A geometry type encodes three components: the reference frame, such as model, world, or view space; the geometric object, such as a point or a direction; and the coordinate scheme, such as Cartesian or

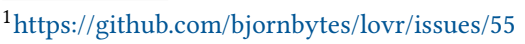

${ }^{2}$ https://github.com/processing/processing/issues/187

${ }^{3}$ https://github.com/JuliaGL/GLVisualize.jl/pull/188
} 
spherical coordinates. Together, these components define which geometric operations are legal and how to implement them.

The core contribution of this paper is the insight that all three components of geometry types are necessary. The three aspects interact in subtle ways, and real-world graphics rendering code varies in each component. Simpler systems that only use a single label [Ou and Pellacini 2010] cannot express the full flexibility of realistic rendering code and cannot cleanly support automatic transformations. We show how encoding geometry types in a real system can help avoid and eliminate realistic geometry bugs. We will explore further how these components are defined and interact in Section 3.

We design a language, Gator, that builds on geometry types to rule out coordinate system bugs and to automatically generate correct transformation code. In Gator, programmers can write teapotVertex in world to obtain a representation of the teapotVertex vector in the world reference frame. The end result is a higher-level programming model that lets programmers focus on the geometric semantics of their programs without sacrificing efficiency.

We implement Gator as an overlay on GLSL [The Khronos Group Inc. [n. d.]], a popular language for implementing shaders in real-time graphics pipelines. Most GLSL programs are also valid in Gator, so programmers can easily port existing code and refine typing annotations to improve its safety. We formalize a relevant subset of our geometry type system and show that erasing the resulting geometry types preserves soundness. In our evaluation, we port rendering programs from GLSL to qualitatively explore Gator's expressiveness and its ability to rule out geometry bugs. We also quantitatively compare the applications to standard GLSL implementations and find that Gator's automatic generation of transformation code does not yield significantly slower rendering time than hand-tuned (and geometry type-less) GLSL code.

This paper's contributions are:

- We identify a class of geometry bugs that exist in geometry-heavy, linear-algebra-centric code such as physical simulations and graphics renderers.

- We design a type system to describe latent coordinate systems present in linear algebra computations and prevent geometry bugs.

- We introduce a language construct that builds on the type system to automatically generate transformation code that is correct by construction.

- We implement the type system and automatic transformation feature in Gator, an overlay on the GLSL language that powers all OpenGL-based 3D rendering.

- We experiment with case studies in the form of real graphics rendering code to show how Gator can express common patterns and prevent bugs with minimal performance overhead.

We begin with some background via a running example before describing Gator in detail.

\section{RUNNING EXAMPLE: DIFFUSE SHADING}

This section introduces the concept of geometry bugs via an example: we implement diffuse lighting, a component of the classic Phong lighting model [Phong 1975]. ${ }^{4}$ We assume some basic linear algebra concepts but no background in graphics or rendering.

\subsection{Gentle Introduction to Shader Programming}

Shader programs are code, typically written in C-like languages such as GLSL or HLSL, that runs on the GPU to render a graphics scene. The GPU executes a pipeline of shader programs, where each shader is specialized to transform a certain property of a graphical object. The shader pipeline consists of several stages. The most notable of these stages are the vertex shader, which outputs

\footnotetext{
${ }^{4}$ Appendix A gives a complete GLSL implementation of the Phong model.
} 
the position of each vertex as a pixel, and the fragment shader, which outputs the color of each fragment, which each corresponds to an on-screen pixel.

In graphics, the scene is a collection of objects. The shape of an object is determined by mesh data consisting of position vectors for each vertex, denoting the spatial structure of the object, and normal vectors, denoting the surface orientation at each vertex.

The kind of transformation each graphics shader applies to a graphical object depends on the pipeline stage. We focus on the vertex shader and the fragment shader, the most common user-programmable stages of the graphics pipeline.

\subsection{Diffuse Lighting}

Diffuse lighting is a basic lighting model that simulates the local illumination on the surface of an object. Given a point on an object, the intensity of its diffuse component is proportional to the angle between the position of the light ray and the local surface normal. The diffuse model first computes the direction of the light by subtracting the mesh (surface) position, fragPos, from the light position:

$$
\text { lightDir }=\text { normalize }(\text { lightPos }- \text { fragPos })
$$

We normalize the vector, which preserves the angle but sets the magnitude to 1 . We calculate the resulting diffuse intensity at this fragment as the angle between the incoming light ray and the fragment normal using the vector dot product:

$$
\text { diffuse }=\max (\text { lightDir } \cdot \text { fragNorm }, 0 .)
$$

The max function used here prevents light from passing through the object by rejecting reflection angles greater than perpendicular.

\subsection{Where Things Go Wrong: GLSL Implementation}

To implement the diffuse lighting model, we must write a GLSL shader program that operates on a per-fragment basis. This section shows how this seemingly simple program translates to surprisingly complex code and creates an opportunity for geometry bugs.

GLSL has vector and matrix types, with names like vec3 and mat4, along with built-in vector functions that make an initial implementation of the diffuse component seem straightforward:

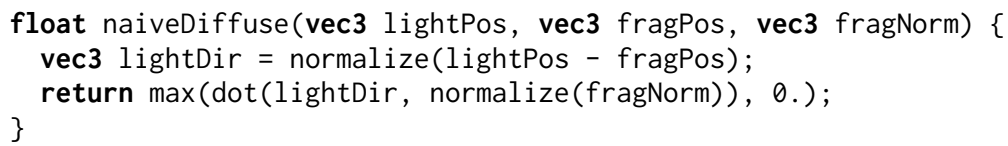

Although lightPos and fragPos have the same type, they are not geometrically compatible: real renderers need to represent them with different reference frames and coordinate schemes. While this code directly reflects the mathematical description above, the output is nonetheless incorrect: it produces the buggy output in Figure $1 \mathrm{~b}$.

Coordinate Systems. The underlying problem is that software needs to represent different vectors in different coordinate systems. Information needed to render the shape of a single graphical object, the positions and normal vectors, lies in the object's model space, as is seen in Figure 2. The definitions of model, world, and view space are as explained in Section 1.1.

Mesh data is scene independent, so we represent mesh parameters such as fragPos and fragNorm initially in model space, independent of the object's current relative position within the scene. In contrast, we represent the position of a light source relative to the entire scene-so lightPos is in world space. As a result, the subtraction expression lightPos - fragPos attempts to compare 
vectors represented in different spaces, yielding a geometrically meaningless result. This bug produces the incorrect output seen in Figure 1b.

Transformation Matrices. To fix this program, the shader needs to transform the two vectors to a common coordinate system before subtracting them. Mathematically, coordinate systems define an affine space, and thus geometric transformations on coordinate systems can be linear or affine. Linear transformations affect only the basis vectors, which can represent rotation and scale, while affine transformations can change both the origin and basis vectors, which can additionally represent translation.

These geometric transformations are represented in code as transformation matrices. To apply a transformation to a vector, shader code uses matrix-vector multiplication. For example, the shader application may provide a matrix uModel that defines the transformation from model to world space using matrix multiplication:

vec3 lightDir $=$ normalize $($ lightPos - uModel * fragPos $))$;

Homogeneous Coordinates. Unfortunately, our current matrix multiplication implementation introduces another bug. Since a $3 \times 3$ Cartesian transformation matrix on 3-dimensional vectors can only express linear transformations, graphics software typically uses a second kind of coordinate system called homogeneous coordinates. An $n$-dimensional vector in homogeneous coordinates uses $n+1$ values: the underlying Cartesian coordinates and a scaling factor, $w$. A $4 \times 4$ transformation matrix in homogeneous coordinates can express affine transformations on the underlying 3-dimensional space, including translation.

Transforming fragPos from model to world space requires both a linear scaling and rotation transformation and a translation to account for change of origins-in other words, an affine transformation matrix. This is a problem: we must represent affine transformation matrix for 3D vectors in homogeneous coordinates-as a $4 \times 4$ matrix. To multiply this matrix by fragPos (which is a 3-dimensional vector), we need a sensible representation of fragPos as a 4-dimensional vector. It is thus not immediately clear by what vector we need to multiply:

vec3 lightDir $=$ normalize $($ lightPos - vec3 (uModel $*$ ?));

To convert from Cartesian to homogeneous coordinates, a vector $[x, y, z]$ becomes $[x, y, z, 1$.$] ; in$ the opposite direction, the homogeneous vector $[x, y, z, w]$ becomes $[x / w, y / w, z / w]$. Thus, to fix our example to use the 4-dimensional affine transformation uModel, we can extend fragPos into a homogeneous vec4 value:

vec3 lightDir $=$ normalize $($ lightPos $-\operatorname{vec} 3($ uModel $* \operatorname{vec} 4($ fragPos, 1.$)))$;

The GLSL functions vec4 and vec3 extend a 3-dimensional vector with the given component and truncate a 4-dimensional vector, respectively. We now have a lightDir in a consistent coordinate system, namely in the world space.

Positions and Normals. The final calculation of the diffuse intensity uses this expression: $\max (\operatorname{dot}($ lightDir, normalize(fragNorm)), 0.$)$

Here, fragNorm resides in model space, we should transform it into world space. One tricky detail, however, is that fragNorm denotes a direction, as opposed to a position as in fragPos. These require different geometric representations, because a direction should not be affected by translation. Fortunately, there is a trick to avoid this issue while still permitting the use of our homogeneous coordinate representation. By extending fragNorm with $w=0$, we do not apply affine translation. return max (dot(lightDir, normalize $(\operatorname{vec} 3($ MModel * vec4(fragNorm, 0.$))))$ ); 
This subtle difference is a common source of errors, particularly for novice programmers. Finally, we have a correct GLSL implementation of diffuse. This version results in the correct output in Figure 1a.

\section{GEOMETRY TYPES}

The problems in the previous section arise from the gap between the abstract math and the concrete implementation in code. We classify this kind of bug, when code performs geometrically meaningless operations, as a geometry bug. Gator provides a framework for declaring a type system that can define and catch geometry bugs in programs.

The core concept in Gator is the introduction of geometry types. These types refine simple GLSL-like vector data types, such as vec3 and mat4, with information about the geometric object they represent. A geometry type consists of three components:

- The reference frame defines the position and orientation of the coordinate system. A reference frame is determined by its basis vectors and origin. Examples of reference frames are model, world, and projective space.

- The coordinate scheme describes a coordinate system by providing operation and object definitions, such as homogeneous and Cartesian coordinates. Coordinate schemes expresses how to represent an abstract value computationally, including what the underlying GLSL-like type is.

- The geometric object describes which geometric construct the data represents, such as a point, vector, direction, or transformation.

In Gator, the syntax for a geometry type is scheme<frame>.object. This notation evokes both module members and parametric polymorphism. Coordinate schemes are parameterized by a reference frame, while geometric objects are member types of a parameterized scheme. For example, cart $3<$ world $>$. point is the type of a point lying in world space represented in a 3D Cartesian coordinate scheme.

The three geometry type components suffice to rule out the errors described in Section 2. The rest of the section details each component.

\subsection{Reference Frames}

We enhance the mathematical definition of diffuse light computation above with geometry types:

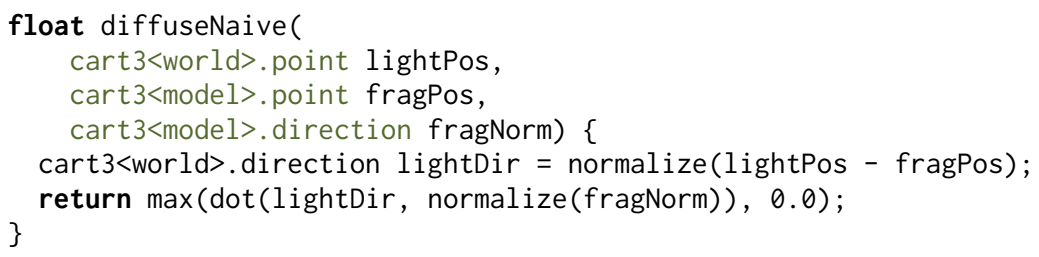

With these stronger types, the expression lightPos - fragPos in this function is an error, since lightPos and fragPos are in different frames. It is geometrically legal to subtract two positions to produce a vector; the only issue with this code is the difference of reference frames. We will further discuss how Gator determines subtraction is legal in Section 3.2.

Definition. Reference frames in Gator are labels with an integer dimension. The dimension of a frame specifies the number of linearly independent basis vectors which make up the frame. Gator does not require explicit basis vectors for constructing frames; keeping basis vectors implicit helps minimize programming overhead and avoid cluttering definitions with unnecessary information. 
We will discuss how transformations between reference frames behave while keeping basis vectors implicit in Section 4.

The Gator syntax to declare the three-dimensional model and world frames is:

frame model has dimension 3 ;

frame world has dimension 3 ;

\subsection{Coordinate Schemes}

To transform fragPos and fragNormal to the world reference frame, we need to provide an affine transformation matrix uModel.

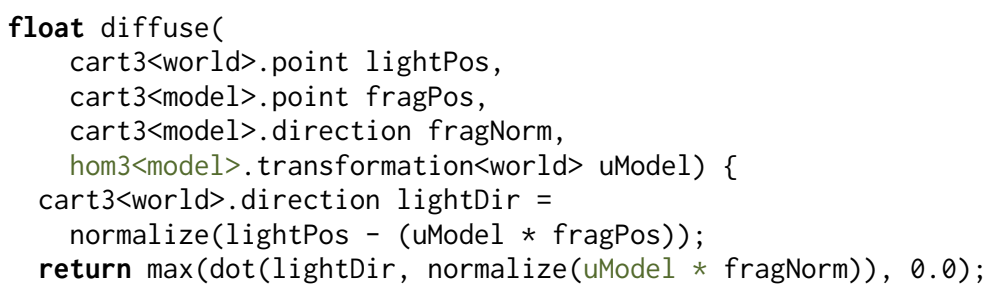

For this example, we define matrix-vector multiplication $m * v$ as applying to types akin to function application: it ensures that $m$ is a transformation in the same frame as the vector and parameterized on the destination frame $f$, then produces an output direction in the frame $f$. With this definition, multiplying uModel by an object in the model reference frame results in an object in the world frame.

Unfortunately, multiplying uModel * fragPos produces a Gator type error since uModel and fragPos are in different coordinate schemes. We will resolve this issue in the next subsection by converting between schemes.

Definition. Coordinate schemes provide definitions of geometric objects and operations. Concretely, they consist of operation type declarations and concrete definitions for member objects and operations. Users are responsible for providing geometrically correct implementations of operations. Recall that, instead of "baking in" a particular notion of geometry, Gator lets coordinate schemes provide types that define correctness for a given set of geometric operations. For example, this code declares a Cartesian coordinate scheme with a vector type:

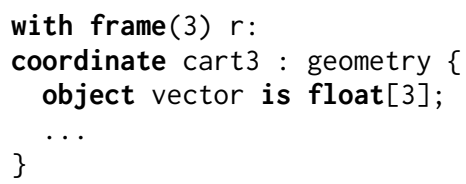

For example, we can define 3D vector addition in Cartesian coordinates, which consists of adding the components of two vectors together:

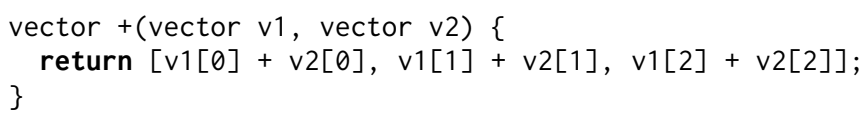

We require all coordinate schemes to be parameterized with reference frames, so cart $3<\operatorname{model}>$ and cart $3<$ world $>$ are different instantiations of the same scheme. Gator's with syntax provides parametric polymorphism in the usual sense; in this example, the 3-dimensional Cartesian coordinate scheme is polymorphic over all 3-dimensional reference frames. Per this definition, if we give coordinate schemes a reference frame of the incorrect dimension, Gator produces a static error. 


\subsection{Geometric Objects}

To apply the uModel affine transformation to our position and normal, we first need to convert each to homogeneous coordinates. Recall from Section 2.3, however, that this coordinate system transformation differs for points and directions. To capture this distinction, we introduce the overloaded function homify: ${ }^{5}$

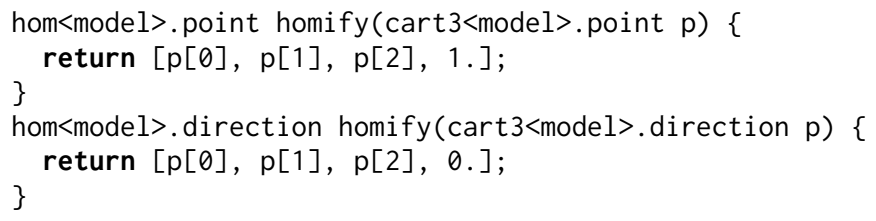

Unlike Cartesian coordinates, homogeneous coordinates have different representations for points and directions: the latter must have zero for its last coordinate, $w$.

To send fragPos and fragNorm to homogeneous coordinates, it suffices to call homify and let the Gator compiler select the correct overloaded variant:

homify(fragPos); // Extends fragPos with w=1. homify(fragNorm); // Extends fragNorm with $w=0$.

We repeat this process to define the function reduce, which maps homogeneous to Cartesian coordinates. Finally, we apply these functions to our model:

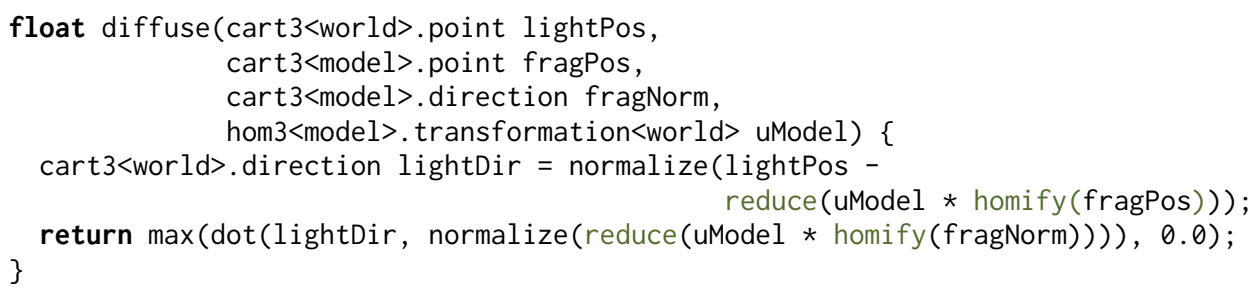

Now, by using all three components of the geometry type, our code will compile and produce the correct Phong diffuse color shown in Figure 1a.

Definition. The object component of a geometry type describes the type's underlying datatype and provides information on permitted operations. Object type definitions can be parameterized on reference frames, such as when writing affine transformations to a specific frame. For example, we can define some objects in homogeneous coordinates:

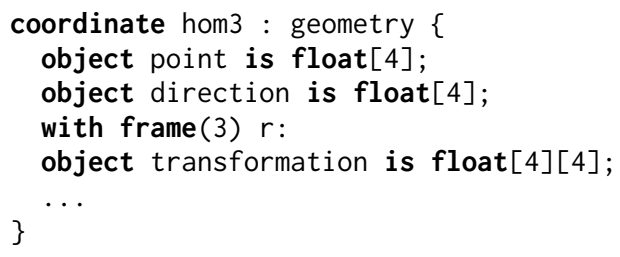

Types and functions declared in coordinate schemes are available in the scope the coordinate scheme is declared. Accessing these functions and types amounts to referencing the coordinate scheme with dot notation (for example, hom3<model>. point refers to point above).

Subtyping in Gator. Object and type declarations in Gator extend existing types; for example, point is defined as a subtype of float[4]. Gator supports a small set of primitives in its core language, such as int, float, and arrays (e.g., float[4]). All types must be given a supertype, which

\footnotetext{
${ }^{5}$ For simplicity, this example homify is written only for objects in the model frame. Gator supports function parameterization on reference frames, so we would normally write homi fy to work on any frame.
} 
may be a geometry type, built-in primitive, or custom type. For example, in the following code, angle is a subtype of float, and both obtuse and acute are subtypes of angle:

type angle is float;

type acute is angle;

type obtuse is angle;

Of special note is how literal values in Gator interact with this subtyping relation: Gator can lift a literal value to any subtype associated to its primitive type. For instance, in this example, we consider a floating-point literal expression (e.g., 3.14) to be a value of type angle, acute, and obtuse. We will examine the exact type behavior of this relationship in detail in Section 5.

When we apply an operation to one or more geometry objects, Gator requires that they have matching coordinate schemes and that the function being applied has a definition in this matching scheme. For example, by omitting a definition for addition between points and their supertypes, we ensure that Gator will reject fragPos + fragPos with a static error.

\section{AUTOMATIC TRANSFORMATIONS}

Gator's type system statically rules out bad coordinate system transformation code. In this section, we show how it can also help automatically generate transformation code that is correct by construction. The idea is to raise the level of abstraction for coordinate system transformations so programmers do not write concrete matrix-vector multiplication computations-instead, they declaratively express source and destination spaces and let the compiler find the right transformations. A declarative approach can obviate complex transformation code that obscures the underlying computation and can quickly become out of date, such as this shift from model to world space:

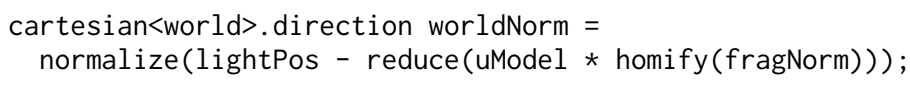

We extend Gator with an in expression that generates equivalent code automatically: cartesian<world $>$.direction worldNorm = normalize(lightPos - fragNorm in world);

The new expression converts a vector into a given representation by generating the appropriate function calls and matrix-vector multiplication. Specifically, the expression $e$ in scheme $<$ frame $>$ takes a typed vector expression $e$ from its current geometry type $T$. object to the type scheme<frame $>$. object by finding a series of transformations that can be applied to $e$. With this notation, either scheme or frame can be omitted without ambiguity, so writing $\mathrm{x}$ in world where $\mathrm{x}$ is in scheme cart3 is the same as writing $x$ in cart3<world $>$. Similarly, writing homPos in cart3 where homPos has reference frame model is the same as writing hompos in cart $3<$ model $>$.

We can only use Gator's in expressions to change the coordinate scheme or parameterizing reference frame; i.e., the geometric object of the target type must be the same as the original type.

Implementation. The Gator compiler implements in expressions by searching for transformations to complete the chain from one type to another. It uses a transformation graph where the vertices are types and the edges are transformation matrices or functions. Figure 3 gives a visual representation of a transformation graph.

\subsection{Canonical Functions}

The transformations that Gator reasons about for automatic application are special: they must uniquely define a map from their domain to their range. Gator requires these functions to be labeled with the keyword canon. Gator defines three requirements on these transformations: (1) there can be only one canonical function between each pair of types in a given scope, (2) all canonical functions between reference frames must map between frames of the same dimension, and (3) a canonical function can only have one non-canonical argument. 


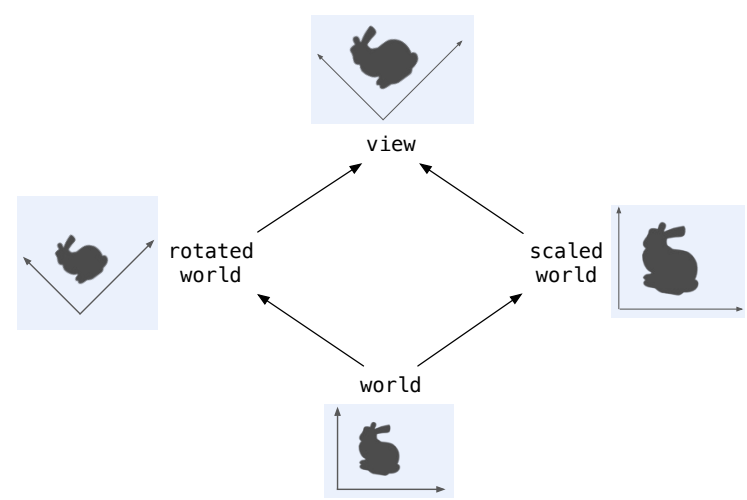

Fig. 3. A transformation graph with provided transformations.

To expand on condition (3); canonical functions may take in canonical arguments, which are variables labeled with the canon keyword. Our familiar example of this use is defining matrix-vector multiplication to be canonical; the matrix itself must be included and must be a canonical matrix:

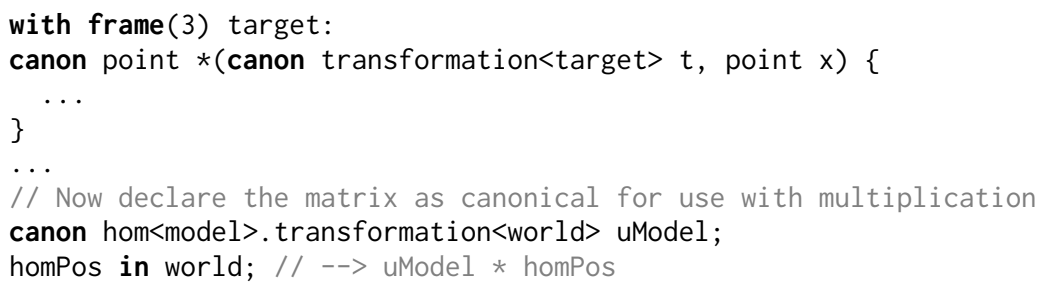

It is also possible to manually invoke canonical functions, in which case the canon annotation is irrelevant. In this sense, canonical functions in Gator resemble automatic type coercions in languages like $\mathrm{C}++$ and $\mathrm{C} \#$.

The intuition of canonical functions comes from affine transformations between frames and coordinate schemes. Since each frame has underlying basis vectors, transformations between frames of the same dimension which preserve these frames are necessarily unique; further, applying these bijective transformations does not cause data to "lose information." Similarly, coordinate schemes simply provide different ways to view the same information; there are often unique transformations between schemes that can be applied as needed to unify data representation.

Canonical function restrictions. Canonical functions must follow some restrictions. First, canonical functions can only be used in the scope they are defined-for this reason, the transformation graph can "lose" edges when a scope ends. Second, overloaded canonical functions are distinct; each "version" of the function is completely separate, and each must follow the conditions outlined above. Finally, the condition that there can be only one canonical function between each pair of types interacts intuitively with subtypes: only one function can be defined between a type and its supertype (in other words, only one canonical function can map between each type with a common subtyping relation). The one exception to this last point is how canonical functions interact with literal types: in expressions can never be applied to expressions with a literal type (e.g., $[1,2,3]$ in world is ambiguous and disallowed), and canonical functions can never take in arguments or produce results of a literal type. 


$$
\begin{aligned}
c & \in \text { constants } \\
x & \in \text { variables } \\
f & \in \text { function names } \\
p & \in \text { primitives } \\
t & \in \text { types }
\end{aligned}
$$

$$
\begin{aligned}
& \tau::=\text { unit }\left|\top_{p}\right| \perp_{p} \mid t \\
& e::=x|c| f\left(e_{1}, e_{2}\right) \mid e \text { as! } \tau \mid e \text { in } \tau \\
& C::=\tau x=e \mid e \\
& P::=C ; P \mid \epsilon
\end{aligned}
$$

Fig. 4. Formal high-level language syntax.

\subsection{Correctness of Generated Transformations}

With in expressions, Gator programmers sacrifice control for convenience: the compiler picks which transformation functions and matrices to use to get from one coordinate system to another. If all the individual transformations marked with canon are correct, then the composed "chain" generated for an in expression must also be correct. Functional verification of transformations, however, is not feasible in Gator's purely static setting: it would require not only the value of every transformation matrix, which typically varies dynamically over time, but also an intrinsic description of each coordinate system, such as the basis vectors for every reference frame, which is rarely available in real graphics code. We view heavyweight dynamic debugging aids for checking transformation correctness as important future work.

We can, however, state a simple consistency condition that is necessary but not sufficient for a system of canonical transformations to be correct. The transformation system should be path independent: for any two types $\tau_{1}$ and $\tau_{2}$, the behavior of any chain of transformations from $\tau_{1}$ to $\tau_{2}$ should be equivalent. In other words, every edge in the transformation graph corresponds to a function-so every path corresponds to a function composition, and every such path between the same two vertices should yield the same composed function. (This definition is equivalent to commutativity for diagrams [Kabra et al. 2020].) Otherwise, the semantics of an expression $e$ in $\tau$ would depend on the graph search algorithm that Gator uses to find routes in the transformation graph, which is clearly undesirable.

However, path independence motivates Gator's requirement that canonical transformations preserve dimensionality (see Section 4.1). Without this condition, we have found it is easy to accidentally violate path independence with non-invertible functions and result in an ambiguous transformation graph for in expressions.

\section{TYPE-DIRECTED TRANSLATION SEMANTICS}

Gator provides a framework for defining geometry types as an "overlay" on top of computationoriented programs in a base language without geometry types. In this section, we formalize a simple model of such an overlay type system. We formalize a general surface language with an extended type system (such as Gator), a general target language with a core set of types (such as GLSL), and a type-directed translation that compiles from the surface language to the core language. We prove a theorem stating that typability is preserved across the translation. The aim is not to model all of Gator; we omit the specifics of coordinate schemes and reference frames in favor of simple types with subtyping, and we omit features such as F-bounded polymorphism where Gator matches the semantics of mainstream languages.

This section defines two languages: a high-level language that includes Gator-style user-defined types, and a low-level abstract target language, Hatchling. Hatchling represents a sound imperative language with an arbitrary set of primitive types and operators on those types. For example, an instance of Hatchling with fixed-size vector and matrix types can reflect a simple core of GLSL. 


$\begin{array}{cccc}\frac{\tau_{1} \leq \tau_{2} \quad \Gamma \vdash e: \tau_{1}}{\Gamma \vdash e: \tau_{2}} & \frac{\mathrm{X}(c)=p}{\Gamma \vdash c: \perp_{p}} \quad \frac{\Gamma(x)=\tau}{\Gamma \vdash x: \tau} & \frac{\Gamma \vdash e: \tau}{\Gamma \vdash \tau x=e: \text { unit }} \\ \frac{\Gamma \vdash C: \tau_{1} \quad \Gamma \vdash P: \tau_{2}}{\Gamma \vdash C ; P: \text { unit }} & \overline{\Gamma \vdash \epsilon: \text { unit }} & \frac{\Gamma \vdash e: \top_{p} \quad \tau \leq \top_{p}}{\Gamma \vdash e \text { as! } \tau: \tau} \\ \frac{\Gamma \vdash e: \tau_{1} \quad A\left(\tau_{1}, \tau_{2}\right)=f}{\Gamma \vdash e \text { in } \tau_{2}: \tau_{2}} & \frac{\Gamma \vdash e_{1}: \tau_{1}}{\Gamma, \vdash e_{2}: \tau_{2} \quad \Phi\left(f, \tau_{1}, \tau_{2}\right)=\tau_{3}} \\ & \frac{\Gamma \vdash f\left(e_{1}, e_{2}\right): \tau_{3}}{\Gamma}\end{array}$

Fig. 5. Typing judgment.

\subsection{Syntax}

Figure 4 lists the syntax of the high-level language we formalize in this section. The types in this core language consist of unit and a lattice over each primitive type $p$. The choice of primitives is kept abstract in this formalism to highlight that the full Gator language applies over arbitrary underlying datatypes. For example, in a GLSL core language, we would have primitive types float and vec3, but a declared type such as vector would be a custom type $t$ and not a primitive $p$.

The core Gator syntactic categories are types $\tau$, expressions $e$, commands $C$, and programs $P$. A program in Gator is a series of commands; we simplify these to variable declarations and expressions. Commands may be raw expressions, so a function call $f\left(e_{1}, e_{2}\right)$ is a valid command. Gator expressions consist of function applications, with as and in expressions to help manage types. We assume functions always take two arguments for simplicity.

\subsection{Typing Rules}

We define a typing judgment for programs in the high-level language, $\Gamma \vdash P: \tau$, that, for program $P$ and typing context $\Gamma$, produces a type $\tau$. The complete semantics for this judgment can be seen in Figure 5. Note that $\Gamma$ is kept constant throughout; declaring a variable requires looking up into the constant $\Gamma$ to determine if the declared type matches the expected type. Keeping $\Gamma$ constant will later help with translation; the type of any expression can be determined exactly from the constant global contexts $\Gamma, \mathrm{X}$, and $\Phi$ along with the judgment $\mathrm{P}$.

Types in the high-level language correspond to a disjoint union of a collection of bounded lattices, one for each primitive type, such that user-defined types are distinct from the bounds on each lattice. We define a type ordering among types $\leq$ where $t_{1} \leq t_{2}$ means that $t_{1}$ is a subtype of $t_{2}$. $\leq$ is reflexive and transitive. In a well formed program, $\leq$ must contain a rule for every user defined type, every type (except unit) must be a subtype of a primitive top type, and every bottom type $\perp_{p}$ must be a subtype of each subtype of the associated $T_{p}$. In other words, $\leq$ must conform to a lattice structure for each primitive $p$. User-defined aliases of primitive type $p$ are bounded by $\top_{p}$ and $\perp_{p}$. Illustrating this structure on our earlier example from Section 3.3, we have $\perp_{\text {float }} \leq$ acute $\leq$ angle $\leq T_{\text {float }}$, and similarly for obtuse. The complete subtyping rules can be found in the attached supplementary materials.

The typing information for functions is stored in a function typing context, $\Phi$, which maps the tuple of function name and input types to the output type; overloaded functions are supported.

This high-level language is parameterized over primitive types stored in primitive type context $X$, which maps literals to their primitive types. 


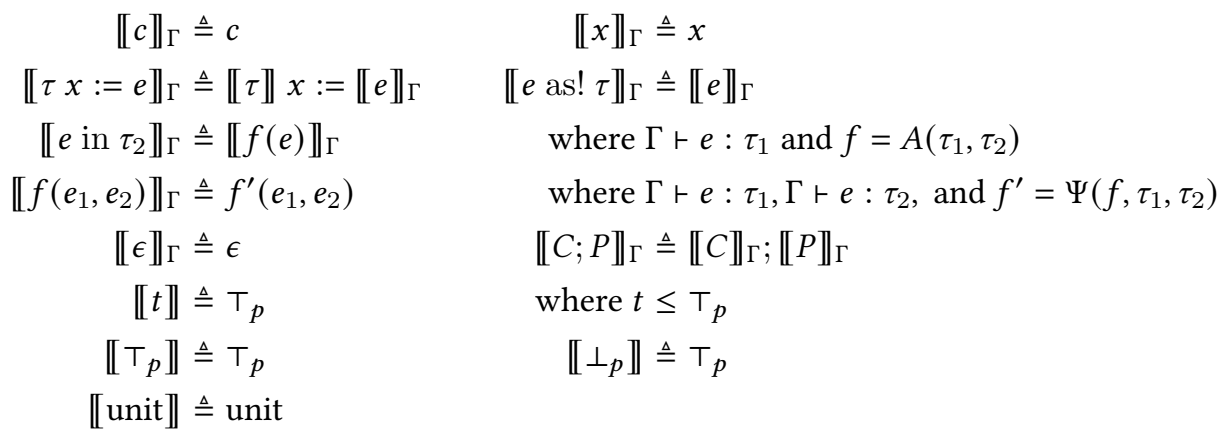

Fig. 6. Translational semantics for expressions and types.

A map $A$ manages the implementation of in expressions. Specifically, $A$ maps a given start and end type $\tau_{1}$ and $\tau_{2}$ to a function name that, when applied to an expression of type $\tau_{1}$, produces an expression of type $\tau_{2}$. We simplify $A$ here to only allow one "step" for notational clarity; in the real Gator implementation, the transformation may be a chain of functions. The exact details of this judgment $A$ are omitted for clarity, but amount to a simple lookup through the available functions for a function of the correct type.

\subsection{Translation Soundness}

To prove the translation soundness of the high-level language, we need to first define Hatchling and our translation from the surface language to it. We will show that a well-typed surface-language program must translate to a well-typed Hatchling program.

We define the syntax of Hatchling based on the surface language, with some features removed. First, Hatchling only has primitive types. We name types in Hatchling such that $T_{p}$ in the surface language translates to $\top_{p}$ in Hatchling. The grammar rule for $\tau$ is $\tau::=$ unit $\mid \top_{p}$. Hatchling also omits as! and in expressions. In other words, Hatchling is simply the surface language with custom type labels and associated operations erased.

Like the surface language, Hatchling is an abstract language. A context $\Xi$ maps an operator name and two input types (for function overloading) to an output type. When Hatchling is instantiated to model a simple core of GLSL, some top types we might see are the float and vec3 types. Translation from Gator would consist of erasing custom geometry types, such as cart3<model>. point, to their associated top type: in this case, vec3.

To translate functions in the surface language (which may be overloaded on types not part of Hatchling), we invoke the context $\Psi$. $\Psi$ maps a function name and input types to a function in the target language. For example, we might map Gator's definition of subtraction between points to be a GLSL subtraction between two vec3s. The resulting function names must each be unique and preserve the translation of primitive types in the surface language. A well formed function translation context $\Psi$ would necessarily map functions to expressions of the correct return type, as constrained by $\Phi$ under translation.

We reuse the judgment $A$ as a mechanism to resolve in expressions, applying the function result of evaluating the judgment to $e$. This must produce a result of the correct translated type for a well-formed judgment $A$. 
The typing rule for operation expressions is:

$$
\frac{\Gamma \vdash e_{1}: \tau_{1} \quad \Gamma, \vdash e_{2}: \tau_{2} \quad \Xi\left(o, \tau_{1}, \tau_{2}\right)=\tau_{3}}{\Gamma \vdash o\left(e_{1}, e_{2}\right): \tau_{3}}
$$

We emphasize that this rule is similar to the surface language's rules for operations, but with a "translated" context using only Hatchling (i.e., primitive) types.

We define translational semantics from the surface language to Hatchling in Figure 6. The typing contexts $\Gamma$ and $\Phi$ are translated by replacing every $\tau$ in their range with $\llbracket \tau \rrbracket$. The main theorem states that these rules translate well-typed programs to well-typed programs:

Theorem 1 (translational soundness). For all $\Gamma, e$, and $\tau$, if $\Gamma \vdash e: \tau$ then $\llbracket \Gamma \rrbracket \vdash \llbracket e \rrbracket_{\Gamma}: \llbracket \tau \rrbracket$.

We provide a proof sketch of this theorem in this paper's accompanying supplementary material. The proof proceeds by structural induction over commands in the surface language.

\section{IMPLEMENTATION}

We implemented Gator in a compiler that statically checks user-defined geometric type systems as described in Section 3 and automatically generates transformation code as described in Section 4 . The compiler consists of 2,800 lines of OCaml. It can emit either GLSL or TypeScript source code, to target either GPU shaders or CPU-side setup code, respectively.

The rest of this section describes how the full Gator language implementation extends the core language features to enable real-world graphics programming. We demonstrate these features in detail in a series of case studies in Section 7.

\subsection{Practical Features}

Types. While Gator is designed around geometry types, writing realistic code requires a more complete language design. Aside from the primitive types bool, int, float, and string, Gator supports fixed-length array types, such as float [3], and type aliases.

New types may be declared as a subtype of an existing type. For instance, we can add support for the GLSL-style vec3:

type vec3 is float[3];

Through creating a custom type alias, we can, for example, provide support for a subtype of float [3], the GLSL vec3. While the built-in float[3] type does not support vector addition, we can write $x+y$ for vec3s $x$ and $y$ as in GLSL.

To allow literal values to interact intuitively with custom types, literals in Gator have special types. For example, the literal expression 42 has type \%int. Gator introduces a typing rule where each literal type $\% p$ is a subtype of every subtype of $p$. In other words, the literal type $\% p$ is the bottom type for the type hierarchy with top type $p$. We summarize these ideas in this example:

type vec 3 is float[3];

vec3 $\mathrm{s} 1=[4.2,4.2,4.2] ; / /$ Legal

float $[3] x=s 1$; // Legal

vec3 s2 = x; // ERROR: float[3] is not a vec3

This behavior of literal values allows us to capture the Gator-style intuition that a given vector can either be a geometric point or just a raw GLSL vec3, but this information is not known until the data is assigned to a variable.

Type Inference. Gator supports local type inference using the auto keyword:

cart3<model>. point fragPos $=\ldots$;

// worldPos will have type cart3<world>. point

auto worldPos = fragPos in world; 
Parametric Polymorphism. Parametric polymorphism in Gator works as presented in a variety of standard languages. Gator types can be parameterized either on Gator types or frames. Parameterizing on a type also allows all subtypes; for instance, the type line in the following example can be parameterized on angle, acute, or obtuse:

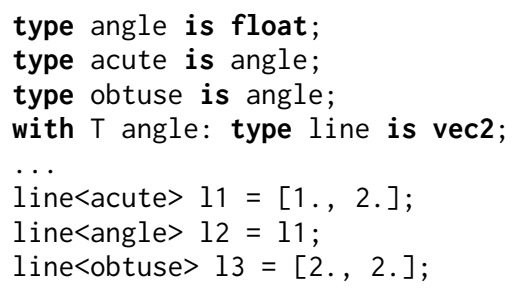

Gator's polymorphic types are covariant, so line<acute $>$ is a subtype of line $<$ angle $>$. This reasoning works similarly for frames and subframes.

External Functions. Functions and variables defined externally in the Gator target language (i.e., GLSL) can be written using the declare keyword.

declare vec3 normalize(vec3 v);

All arithmetic operations in Gator are functions which can be declared and overloaded. Requiring this declaration allows us to include GLSL-style infix addition of vectors without violating coordinate systems restrictions:

declare vec3 +(vec3 v1, vec3 $\vee 2)$;

Addition is then valid for values of type vec 3 :

vec3 $x=[0 ., 1 ., 2$.$] ;$

vec3 result $=x+x ; / /$ Legal

However, addition emits an error when applied to two points, as desired, since they are not subtypes of vec3 and so there is no valid function overload:

cartesian<model>. point fragPos $=[0 ., 1 ., 2$.$] ;$

auto result $=$ fragPos + fragPos; // ERROR: No addition defined for points

Import System. To support using custom Gator libraries, we built a simple import system. Files are imported with the keyword using followed by the filename:

using "../glsl_defs.lgl";

Unsafe Casting. As an escape hatch from strict vector typing, Gator provides an unsound cast expression written with as!:

vec 3 position = fragPos as! vec 3 ;

Casts must preserve the primitive representation; we could not, for instance, cast a variable with type float [2] to float [3]. Unsafe casts syntactically resemble in expressions but are unsound and carry no run-time cost. These casts allow the implementation of known-safe transformations as low-level data manipulations, and they let the user forgo Gator's type system and work directly with GLSL-like semantics, as seen in the example above.

\subsection{Standard Library}

Per Section 5, Gator does not include any built-in functions or operations, which are instead given as external contexts. Our implementation does provide array indexing as a built-in function to help simplify definitions, but otherwise requires that operations such as + be explicitly declared. 
We implement a standard library to provide access to common GLSL operations. This library consists of GLSL function declarations, scheme declarations for Cartesian and homogeneous coordinates, and basic transformation functions such as homify and reduce. We declare relevant GLSL functions to work on GLSL types, such as the addition operation in section 6:

declare vec3 $+(\operatorname{vec} 3 x, \operatorname{vec} 3 \mathrm{y})$;

We build schemes in much the same way as introduced in Section 3.2, as with the sketch of the cart3 scheme:

with frame(3) $r$ :

coordinate cart 3 : geometry \{

object vector is float [3];

vector +(vector $\mathrm{v} 1$, vector $\mathrm{v} 2)\{$ \}

return $[\mathrm{v} 1[0]+\mathrm{v} 2[0], \mathrm{v} 1[1]+\mathrm{v} 2[1], \mathrm{v} 1[2]+\mathrm{v} 2[2]]$ \}

Finally, we include homify and reduce transformations between homogeneous and Cartesian coordinates as discussed in Section 3.3:

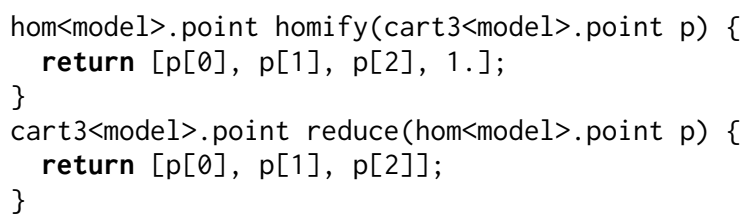

We use this same library when implementing each shader for the case study.

\section{GATOR IN PRACTICE}

This section explores how Gator can help programmers avoid geometry bugs using a series of case studies. We use the Gator compiler to implement OpenGL-based renderers that demonstrate a variety of common visual effects, and we compare against implementations in plain GLSL. We report qualitatively on how Gator's type system influences the expression of the rendering code (Section 7.1) and quantitatively on the performance impact of Gator's in expressions (Section 7.2).

\subsection{Case Studies}

To qualitatively study Gator's safety and expressiveness, we used it to implement 8 renderers based on the OpenGL API in its browser-based incarnation, WebGL [Jackson and Gilbert 2015]. To the best of our knowledge, there is no standard benchmark suite for evaluating the expressiveness and performance of graphics shader programs. Instead, we assemble implementations of a range of common rendering effects:

- Phong: The lighting model introduced in Section 2.

- Reflection: Use two-pass rendering to render an object that reflects its surroundings.

- Shadow map: Simulate shadows for moving objects by computing a projection.

- Microfacet: Texture model for simulating roughness on a surface.

- Texture: Use OpenGL's texture mapping facility to draw an image on the surface of an object.

- Spotlight: Phong lighting restricted to a spotlight circle.

- Fog: Lighting model with integration to simulate distortion from fog.

- Bump map: Texture model for simulating bumps on surfaces.

Each renderer consists of both CPU-side "host" code and several GPU-side shader programs. Figure 7 depicts the output of a selection of these renderers. 


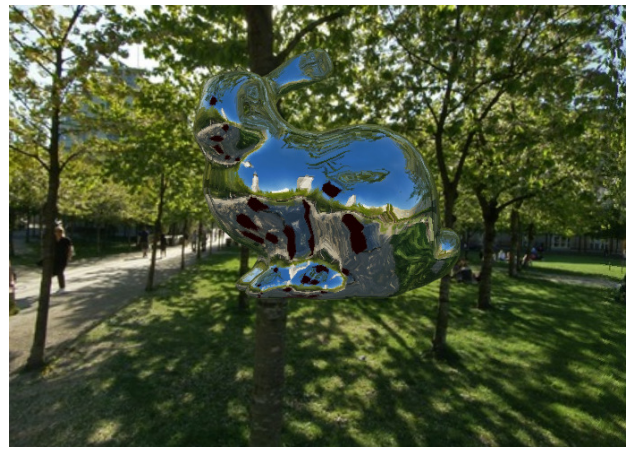

(a) Reflection.

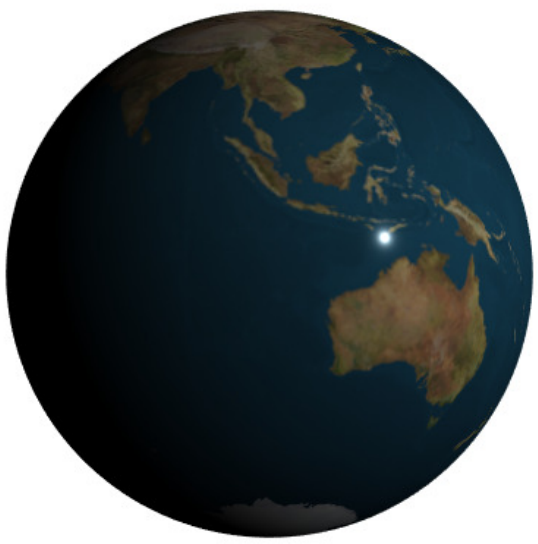

(c) Microfacet.

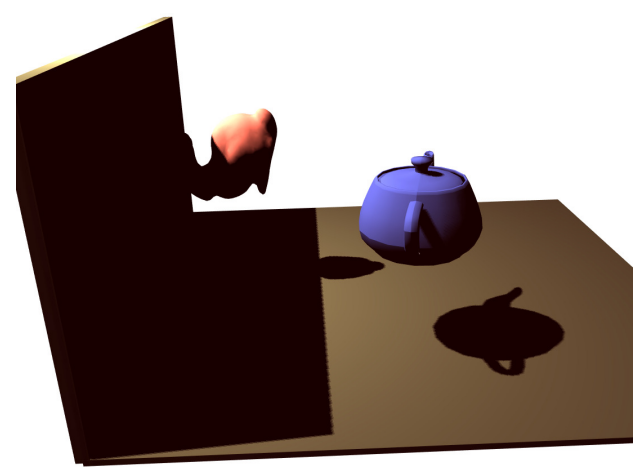

(b) Shadow map.

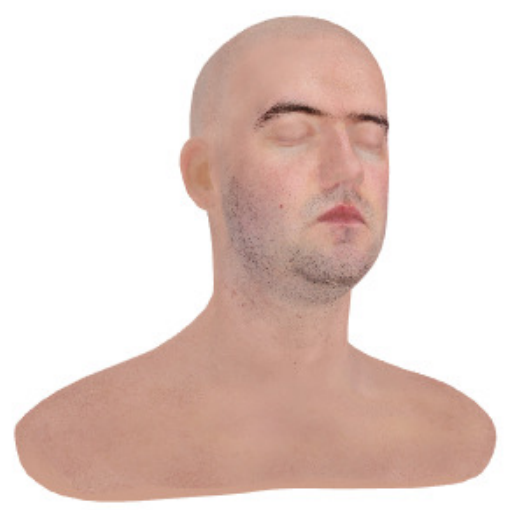

(d) Texture.

Fig. 7. Example outputs from four renderers used in our case studies.

The rest of this section reports on salient findings from the case studies and compares them to standard implementations in GLSL and TypeScript. For the sake of space, we highlight the most distinct cases where Gator helped clarify geometric properties and prevent geometry bugs that would not be caught by plain GLSL. The complete code of both the Gator and reference GLSL implementations is available online. ${ }^{6}$

Reflection. Our reflection case study, shown in Figure 7a, renders an object that reflects the dynamic scene around it, creating a "mirrored" appearance. The surrounding scene includes a static background texture, known as a skybox, and several non-reflective floating objects to demonstrate how the reflected scene changes dynamically.

Rendering a reflection effect requires several passes through the graphics pipeline. The idea is to first render the scene that the mirror-like object will reflect, and then render the scene again with that resulting image "painted" onto the surface of the object. There are three main phases: (1) Render the non-reflective objects from the perspective of the reflective object. This requires six

\footnotetext{
${ }^{6}$ https://github.com/cucapra/gator
} 
passes, one for each direction in 3-space. (2) Render the reflection using the generated cube as a texture reference. (3) Finally, render all other objects from the perspective of the camera.

Reflection: Inverse Transformation. For the second step, we use a cubemap-a special GLSL texture with six sides-to capture the six directions of the scene. To calculate the angle of reflection, we need to reason about the interactions of the light rays in view space as they map onto our model space. Specifically, calculating the reflection amounts to the following operations, where $\mathrm{V}$ is the current vertex position and $\mathrm{N}$ is the current normal vector, which must both be in the view frame: uniform samplerCube<alphaColor > uSkybox;

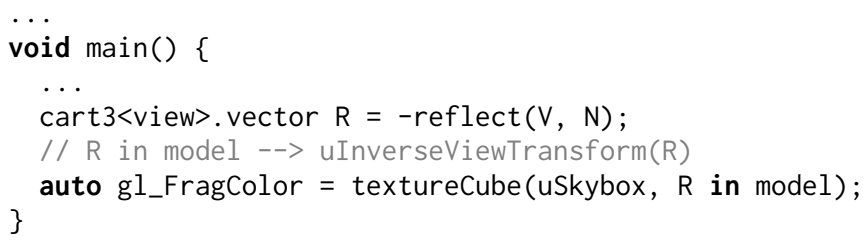

The key feature to note here is the transformation $\mathrm{R}$ in model, which accomplishes our goal of returning the light calculation to the object's perspective (the model frame). This transformation requires that we map backwards through the world frame, a transformation which requires the inverse of the model $\rightarrow$ world matrix and the world $\rightarrow$ view matrix multiplied together. This interaction produces a unique feature in Gator's type system, where we need to have both a forward transformation and its inverse. The shader declares the matrices as follows, with the inversion being done preemptively on the CPU:

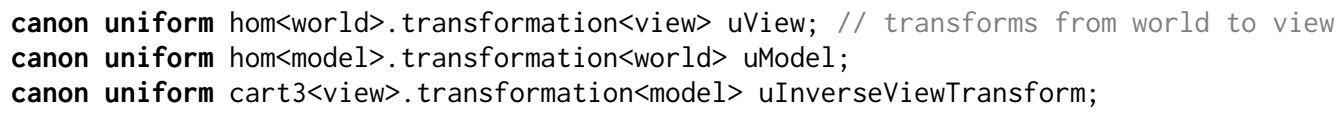

The inverse view transform uses a Cartesian (cart3) matrix because we intend only to use it for the vector $\mathrm{R}$, which ignores the translation component of the affine transformation. The inverse transformation is what permits us to write $R$ in model, while the forward transformations must be uniquely given to actually send our position and normal to the view frame (as noted before).

Reflection: Normal Transformation. Additionally, we need to reason about the correct transformation of the normal with translation (that is, when moving the object in space), which means that we need the inverse transpose matrix, which provides a distinct path between the model and view frames. The use of the inverse transpose of the model-view matrix is perhaps unexpected; it arises specifically for geometry normals from a convenient algebraic equation (which arises from the requirement that the normal and tangent vectors of a matrix must be perpendicular).

In GLSL, it is easy to mistakenly transform the normal as if it were an ordinary direction:

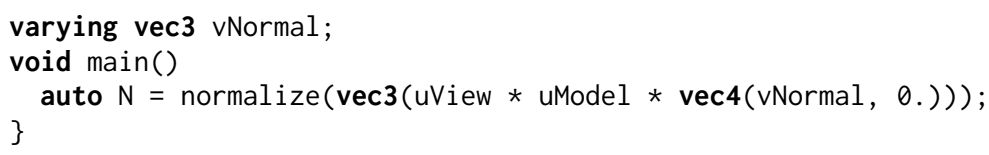

This code is wrong because uModel $* \operatorname{vec} 4(v N o r m a l, 0$.$) does not apply the translation component$ of the uModel transformation. To prevent this kind of bug, the Gator standard library defines the normal type, which is a subtype of vector. A new normalTransformation type can only operate on normals. Using these types, a simple in transformation suffices:

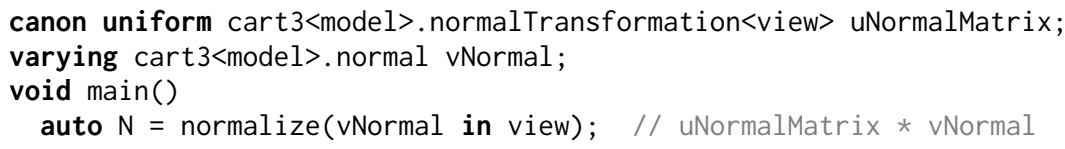




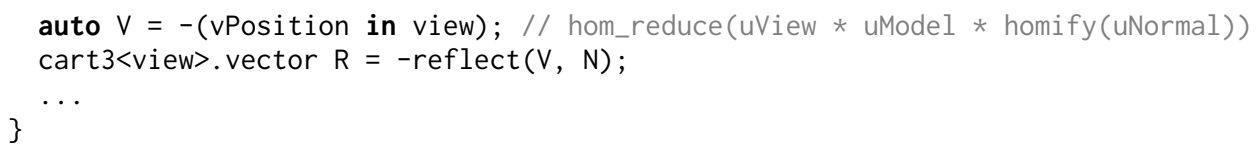

The compiler uses the normal version of the transformation, correctly applying the translation component, allowing a correct computation of $\mathrm{R}$.

Shadow Map: Light Space. Shadow mapping is a technique to simulate the shadows cast by 3D objects when illuminated by a point light source. Our case study, shown in Figure 7b, renders several objects that cast shadows on each other and a single "floor" surface. We simulate the non-shadow coloring through Phong lighting as previously discussed.

As with the reflection renderer, to calculate shadows in a scene, we require several passes through the graphics pipeline. The first pass renders the scene from the perspective of the light and calculates the whether a given pixel is obscured by another. The second pass uses this information to draw shadows; a given pixel is lit only if it is not obscured from the light.

The first pass does all geometric operations in the vertex shader to render from the light's perspective. This is easy to get wrong in GLSL by defaulting to the usual transformation chain:

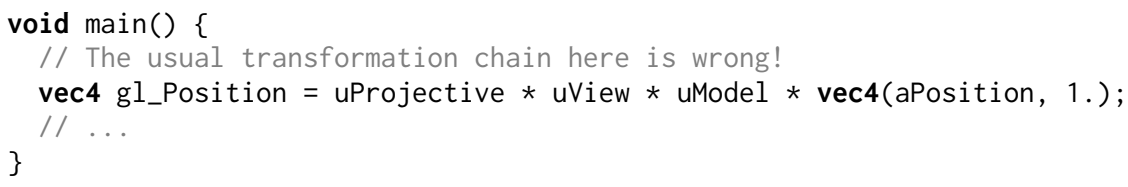

This incorrect transformation will lead to shadows in strange places and hard-to-debug effects.

In Gator, on the other hand, we do the work when typing the matrices themselves. From there, the transformation to light space is both documented and correct by construction:

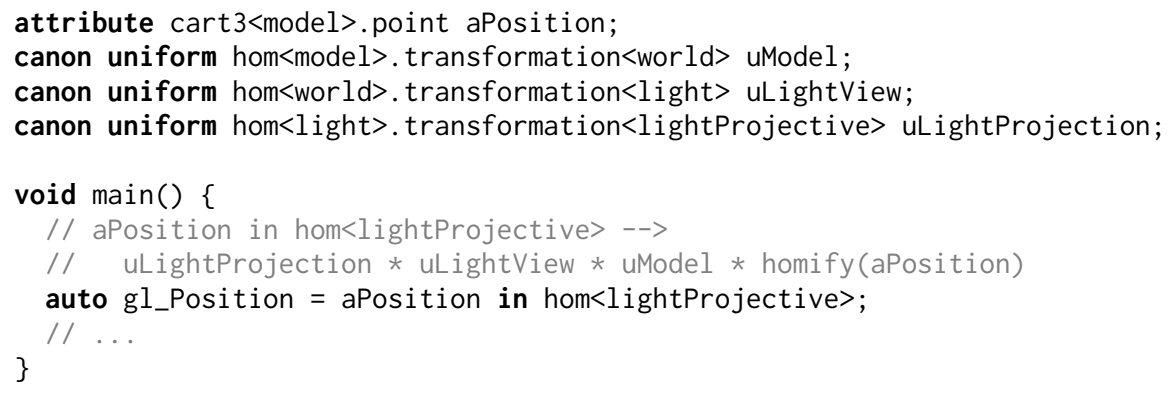

We use the depth information in the final pass in the form of uTexture. To look up where the shadow should be placed, we must look up the position of the current pixel in the light's projective space (which is where the position was represented in the previous rendering). In GLSL, we require the following hard-to-read code:

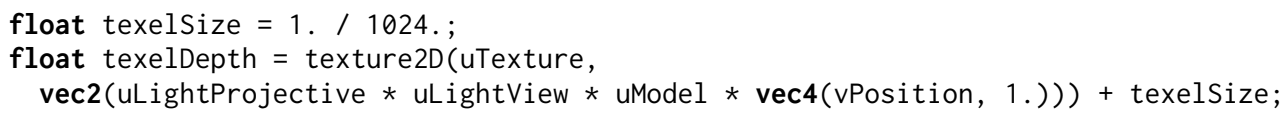

Using the correct transformations is difficult and hard to be sure if the correct transformation chain was used once again. In Gator, on the other hand, this is straightforward:

float texelDepth $=$ texture2D(uTexture, vec2(vPosition in lightProjective) $)+$ texelsize;

Microfacet: Custom Canonical Functions. Anisotropic microfacet shading creates an illusion of roughness and bumpiness on a $3 \mathrm{D}$ modeled surface using information from the normal map of that 
surface. Modeling this correctly, however, requires an unusual technique: building a local reference frame from the perspective of the normal vector called the local normal frame.

Converting to the local normal frame of a given normal consists of a function call with the appropriate normal vector:

vec3 proj_normalframe $(\operatorname{vec} 3 \mathrm{~m}, \operatorname{vec} 3 \mathrm{n})\{\ldots\}$

vec3 geom_normal;

vec3 result $=$ proj_normalframe(viewDir, geom_normal);

However, as with other conversions between spaces, writing this kind of code in GLSL can involve multiple nonobvious steps. If the normal and target direction are in different spaces, the GLSL code must look like this:

vec3 result $=$ proj_normalframe $(\operatorname{vec} 3($ uView $*$ uModel $*$ vec4 $($ modelDir, 1.$))$, geom_normal);

In Gator, we instead declare proj_normalframe with the appropriate type and a canonical tag, noting that the normal itself is a canonical part of the transformation:

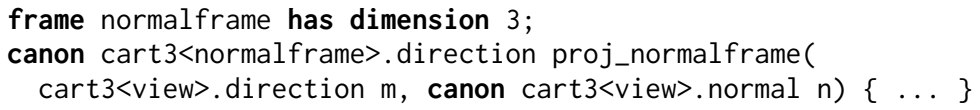

We then declare the normal geom_normal with the appropriate type, and the transformation type becomes straightforward:

canon cart3<view>.normal geom_normal;

auto result $=$ modelDir in normalframe;

Textures: Parameterized Types. A texture is an image that a renderer maps onto the surface of a 3D object, creating the illusion that the object has a "textured" surface. Our texture case study renders a face mesh with a single texture (shown in Figure 7d). While this example does not provide any geometry insight, we highlight the study to show the broad utility of the types introduced by Gator for a graphics context. GLSL represents a texture using a sampler2D value, which acts as a pointer to the requested image, which is typically an input to a shader:

uniform sampler2D uTexture;

Textures are mapped to the image using the object's current texture coordinate:

varying vec2 vTexCoord;

Whereas textures themselves are typically constant (as indicated by the uni form keyword), a texture coordinate vTexCoord differs for each vertex in a mesh (as the varying keyword indicates). To sample a color from textures at a specific location, a fragment shader uses the GLSL texture2D function:

vec4 gl_FragColor = texture2D (uTexture, vTexCoord);

The result type of texture2D in GLSL is vec4: while textures typically contain colors (consisting of red, green, blue, and alpha channels), renderers can also use them to store other data such as shadow maps or even points in a coordinate system.

In Gator and its GLSL standard library, sampler2D is a polymorphic type which indicates what values it contains:

with float[4] $\mathrm{T}$ :

declare type sampler2D;

with float[4] $\mathrm{T}$ :

declare $T$ texture (sampler $2 \mathrm{D}<\mathrm{T}>$ tex, vec2 uv);

For this renderer, the texture contains alphaColor values, which represent colors in gl_FragColor. The Gator fragment shader is nearly identical to GLSL but with more specific types:

uniform sampler2D<alphaColor> uTexture;

varying vec2 vTexCoord; 


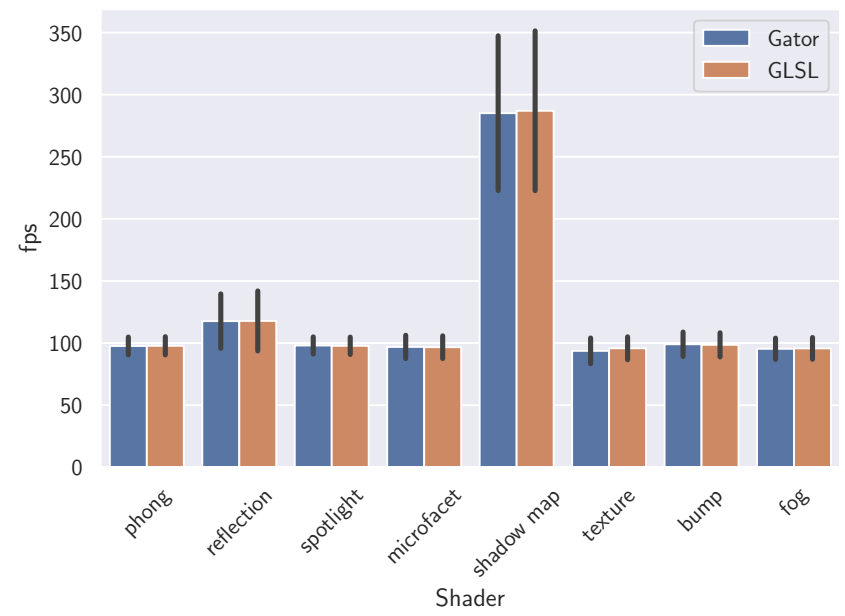

Fig. 8. The mean frames per second (fps) for each shader for both the baseline (GLSL) and Gator code. Error bars show the standard deviation.

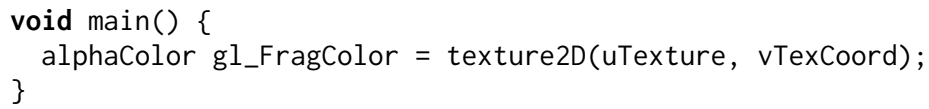

With this code, we guarantee that the texture represented by uTexture produces a color which is assigned into gl_FragColor. We therefore both provide documentation and prevent errors when trying to use the resulting vector as, say, a point for later calculations.

\subsection{Performance}

While Gator is chiefly an "overhead-free" wrapper that expresses the same semantics as an underlying language, there is one exception where Gator code can differ from plain GLSL: its automatic transformation insertion using in expressions (Section 4).

The Gator implementation compiles in expressions to a chain of transformation operations that may be slower than the equivalent in a hand-written GLSL shader. In particular, hand-written GLSL code can store and reuse transformation results or composed matrices, while the Gator compiler does not currently attempt to do so. The Gator compiler also generates function wrappers to enable its overloading. While both patterns should be amenable to cleanup by standard compiler optimizations, this section measures the performance impact by comparing Gator implementations of renderers from our case study to hand-optimized GLSL implementations.

7.2.1 Experimental Setup. We perform experiments on Windows 10 version 1903 with an Intel i7-8700K CPU, NVIDIA GeForce GTX 1070, 16 GB RAM, and Chrome 81.0.4044.138. We run 60 testing rounds, each of which executes the benchmarks in a randomly shuffled order. In each round of testing, we execute each program for 20 seconds while recording the time to render each frame. We report the mean and standard deviation of the frame rate across all rounds.

7.2.2 Performance Results. Figure 8 shows the average frames per second (fps) for the GLSL and Gator versions of each renderer and Table 1 shows mean and standard deviation of each frame rate. The frame rates for the two versions are generally very similar-the means are all within one standard deviation. Several benchmarks have frame rates around $100 \mathrm{fps}$ because they render the same number of objects and the bulk of the cost comes from scene setup. We used around 
Table 1. Mean and standard error of the frame rate for the Gator and GLSL (baseline) implementations. We also give the $p$-value for a Wilcoxon sign rank test and two one-sided $t$-test (TOST) equivalence test that checks whether the means are within $1 \mathrm{fps}$, where * denotes statistical significance $(p<0.05)$.

\begin{tabular}{ccccccccc} 
& \multicolumn{2}{c}{ Gator } & & \multicolumn{2}{c}{ GLSL } & & \multicolumn{2}{c}{$p$-value } \\
\cline { 2 - 3 } \cline { 7 - 8 } Shader & Mean & S.E. & & Mean & S.E. & & Wilcoxon & TOST \\
\hline phong & 97.84 & 0.22 & & 97.67 & 0.21 & & 0.187 & $0.003^{*}$ \\
texture & 95.82 & 0.27 & & 93.75 & 0.31 & & $<0.001^{*}$ & 0.996 \\
reflect & 117.8 & 0.72 & & 117.7 & 0.65 & & 0.638 & 0.188 \\
shadow & 287.0 & 1.91 & & 285.1 & 1.85 & & 0.365 & 0.636 \\
bump & 98.60 & 0.29 & & 99.07 & 0.29 & & 0.063 & 0.098 \\
microfacet & 96.71 & 0.27 & & 96.91 & 0.28 & & 0.640 & $0.020^{*}$ \\
fog & 95.74 & 0.26 & & 95.41 & 0.25 & & 0.119 & $0.033^{*}$ \\
spotlight & 97.83 & 0.21 & & 98.07 & 0.20 & & 0.299 & $0.005^{*}$ \\
\hline
\end{tabular}

Table 2. Instances of language features used in each shader. The Unique Frames column indicates the number of unique frame types used by each benchmark, while the in and as! columns indicate the number of times each kind of expression appears. All counts include both the fragment and vertex shader of each example.

\begin{tabular}{cccc} 
Shader & Unique Frames & in & as! \\
\hline phong & 5 & 5 & 0 \\
texture & 4 & 1 & 0 \\
reflect & 4 & 11 & 0 \\
shadow & 6 & 5 & 0 \\
bump & 5 & 2 & 0 \\
microfacet & 5 & 5 & 7 \\
fog & 5 & 8 & 2 \\
spotlight & 5 & 7 & 0 \\
\hline
\end{tabular}

100 objects for all scenes except reflection and shadow to reduce natural variation and focus on measuring the cost of the shaders.

Table 1 shows the results of Wilcoxon signed-rank statistical tests that detect differences in the mean frame rates. At an $\alpha=0.05$ significance level, we find a statistically significant difference only for texture. However, a difference of means test cannot confirm that a difference does not exist. For that, we also use we use the two one-sided $t$-test (TOST) procedure [Schuirmann 2005], which yields statistical significance $(p<\alpha)$ when the difference in means is within a threshold. We use a threshold of $1 \mathrm{fps}$. The test rejects the null hypothesis-concluding, with high confidence, that the means are similar-for the phong, microfacet, fog, and spotlight shaders.

The anomaly is texture, where our test concludes that a small ( $2 \mathrm{fps})$ performance difference does exist, although the differences are still within one standard deviation. We hypothesize the culprit to be the boilerplate functions inserted by Gator, some of which can be optimized away with more work.

\subsection{Language Constructs}

Table 2 lists the use frequency of some Gator language constructs in the benchmarks. Applications generally use several frames and all use at least one in expression. Most applications do 
not need unsound as! expressions. Where they do appear, they are primarily used to shore up issues surrounding type inference and generics; we expect some could be removed from these standard examples with more compiler engineering effort. Specifically, some instances work around limitations in the way that the type of normal vectors works. Others work around a known bug in the version of the compiler as of this writing where the assignment $b=-b$ fails to typecheck for a Cartesian direction $b$ due to an interaction between subtyping and polymorphic functions in coordinate schemes.

\section{RELATED WORK}

SafeGI [Ou and Pellacini 2010] introduces a type system as a C/C++ library for geometric objects parameterized on reference frame labels not unlike Gator's geometry types. SafeGI's types do not include information about the coordinate scheme, and so also require abstracting the notion of transformations to a map type which must be applied through a layer of abstraction. Additionally, SafeGI does not attempt to introduce automatic transformations like Gator's in expressions nor attempt to study the result of applying these types to real code.

The dominant mainstream graphics shader languages are OpenGL's GLSL [The Khronos Group Inc. [n. d.]] and Direct3D's HLSL [Microsoft 2008]. Research on graphics-oriented languages for manipulating vectors dates at least to Hanrahan and Lawson's original shading language [Hanrahan and Lawson 1990]. Recent research on improving these shading languages has focused on modularity and interactions between pipeline stages: Spark [Foley and Hanrahan 2011] encourages modular composition of shaders; Spire [He et al. 2016] facilitates rapid experimentation with implementation choices; and Braid [Sampson et al. 2017] uses multi-stage programming to manage interactions between shaders. These languages do not address vector-space bugs. Gator's type system and transformation expressions are orthogonal and could apply to any of these underlying languages.

Scenic [Fremont et al. 2019] introduces semantics to reason about relative object positions and $\lambda$ CAD [Nandi et al. 2018] introduces a small functional language for writing affine transformations, although neither seem to have a type system for checking the coordinate systems they have defined. Practitioners have noticed that vector-space bugs are tricky to solve and have proposed using a naming convention to rule them out [Sylvan 2017]. A 2017 enumeration of programming problems in graphics [Sampson 2017] identifies the problem with latent vector spaces and suggests that a novel type system may be a solution. Gator can be seen as a realization of this proposal.

Gator's type system works as an overlay for a simpler, underlying type system that only enforces dimensional restrictions. This pattern resembles prior work on type qualifiers [Foster et al. 1999], dimension types [Kennedy 1994], and type systems for tracking physical units [Kennedy 1997] Canonical transformations in Gator are similar in feel to Haskell's type class polymorphic functions, where Gator's canonical geometry types can be defined as a type class and the in keyword behave similarly to Haskell's lookups. Additionally, Gator's notion of automatic transformations is a specialized use of type coercion, similar to structures introduced in the $\mathrm{C \#}$ and $\mathrm{C}++$ languages. What is particular about Gator's automatic type coercion is the unenforced notion of path independence discussed in Section 4, along with a definition of uniqueness of canonical transformations. Together, these requirements allow automation of coordinate system transformations that would not be allowed in other, similar systems.

\section{CONCLUSION}

Gator attacks a main impediment to graphics programming that makes it hard to learn and makes rendering software hard to maintain. Geometry bugs are extremely hard to catch dynamically, so Gator shows how to bake safeguards into a type system and how a compiler can declaratively generate "correct by construction" geometric code. We see Gator as a foundation for future work 
that brings programming languages insights to graphics software, such as formalizing the semantics of geometric systems and providing abstractions over multi-stage GPU pipelines.

Geometry bugs are not just about graphics, however. Similar bugs arise in fields ranging from robotics to scientific computing. In Gator, users can write libraries to encode domain-specific forms of geometry: affine, hyperbolic, or elliptic geometry, for example. We hope to expand Gator's standard library as we apply it to an expanding set of domains.

\section{ACKNOWLEDGMENTS}

Thank you to Eric Campbell, Jonathan DiLorenzo, Ryan Doenges, Andrew Hirsch, Steve Marschner, Andrew Myers, Rachit Nigam, Rolph Recto, Eston Schweickart, Isaac Sheff, Steffen Smolka, and Alexa VanHattum for reading drafts of our paper and providing valuable feedback. Thank you to Henry Liu and Ben Gillott for providing work on the examples and compiler presented in this paper. This work was supported in part by the Center for Applications Driving Architectures (ADA), one of six centers of JUMP, a Semiconductor Research Corporation program co-sponsored by DARPA. Support also included NSF award \#1845952.

\section{REFERENCES}

Tim Foley and Pat Hanrahan. 2011. Spark: Modular, Composable Shaders for Graphics Hardware. In SIGGRAPH. Jeffrey S. Foster, Manuel Fähndrich, and Alexander Aiken. 1999. A Theory of Type Qualifiers. In ACM Conference on Programming Language Design and Implementation (PLDI).

Daniel J. Fremont, Tommaso Dreossi, Shromona Ghosh, Xiangyu Yue, Alberto L. Sangiovanni-Vincentelli, and Sanjit A. Seshia. 2019. Functional Programming for Compiling and Decompiling Computer-Aided Design. In ACM Conference on Programming Language Design and Implementation (PLDI).

Pat Hanrahan and Jim Lawson. 1990. A Language for Shading and Lighting Calculations. In SIGGRAPH.

Yong He, Tim Foley, and Kayvon Fatahalian. 2016. A System for Rapid Exploration of Shader Optimization Choices. In SIGGRAPH.

Dean Jackson and Jeff Gilbert. 2015. WebGL Specification. https://www.khronos.org/registry/webgl/specs/latest/1.0/.

Aditi Kabra, Dietrich Geisler, and Adrian Sampson. 2020. Online Verification of Commutativity. In Workshop on Tools for Automatic Program Analysis (TAPAS).

Andrew J. Kennedy. 1994. Dimension Types. In European Symposium on Programming (ESOP).

Andrew J. Kennedy. 1997. Relational Parametricity and Units of Measure. In ACM SIGPLAN-SIGACT Symposium on Principles of Programming Languages (POPL).

Microsoft. 2008. Direct3D. https://msdn.microsoft.com/en-us/library/windows/desktop/hh309466(v=vs.85).aspx.

Chandrakana Nandi, James R. Wilcox, Pavel Panchekha, Taylor Blau, Dan Grossman, and Zachary Tatlock. 2018. Functional Programming for Compiling and Decompiling Computer-Aided Design. In ACM SIGPLAN International Conference on Functional Programming (ICFP).

Jiawei Ou and Fabio Pellacini. 2010. SafeGI: Type Checking to Improve Correctness in Rendering System Implementation. In Eurographics Conference on Rendering (EGSR).

Bui Tuong Phong. 1975. Illumination for Computer Generated Pictures. Commun. ACM 18, 6 (June 1975), 311-317.

Adrian Sampson. 2017. Let's Fix OpenGL. In Summit on Advances in Programming Languages (SNAPL).

Adrian Sampson, Kathryn S McKinley, and Todd Mytkowicz. 2017. Static Stages for Heterogeneous Programming. In ACM Conference on Object-Oriented Programming, Systems, Languages, and Applications (OOPSLA).

Donald J. Schuirmann. 2005. A comparison of the Two One-Sided Tests Procedure and the Power Approach for assessing the equivalence of average bioavailability. Journal of Pharmacokinetics and Biopharmaceutics 15 (2005), 657-680.

Mark Segal and Kurt Akeley. 2017. The OpenGL 4.5 Graphics System: A Specification. https://www.opengl.org/registry/doc/ glspec45.core.pdf.

Sebastian Sylvan. 2017. Naming Convention for Matrix Math. https://www.sebastiansylvan.com/post/matrix_naming_ convention/.

The Khronos Group Inc. [n. d.]. The OpenGL ES Shading Language (1.0 ed.). The Khronos Group Inc. 